\title{
Effect of Chlorotriphenyl Derivatives of Sn and Pb upon Biophysical Properties of Membranes
}

\author{
Dariusz Man, ${ }^{1}$ Marian Podolak, ${ }^{1}$ Grzegorz Engel, ${ }^{1}$ and Ewa Boniewska ${ }^{2}$ \\ ${ }^{1}$ Institute of Physics, Opole University, Oleska 48, 45-052 Opole, Poland \\ ${ }^{2}$ Institute of Biotechnology and Molecular Biology, Opole University, ul. Kominka 4, 45-033 Opole, Poland
}

Correspondence should be addressed to Dariusz Man, dariusz.man@uni.opole.pl

Received 11 February 2009; Revised 5 May 2009; Accepted 20 May 2009

Recommended by Rumiana Koynova

Biophysical activity of two twin organometallic compounds Triphenyltin chloride (TPhT) and Triphenyllead chloride (TPhL) in their interreaction with model membranes, as well as with yeast cells Saccharomyces cerevisiae, was investigated. Four measurement methods were used in the experiments: two physical methods (spin probes method and the electric method); two biological methods (minimal inhibitory concentration /MIC/ and yeast survival test). It has been found that the activity of TPhT in interaction with model membranes and yeast cells is distinctly greater than that of TPhL. The activity manifests itself by considerable increase in the fluidity of the middle part of liposome bilayer, change in the polarization direction of the transmembrane voltage of filters impregnated with lauric acid, and in complete inhibition of growth of yeast cells at the concentration of $5 \mu \mathrm{g} / \mathrm{mL}$.

Copyright () 2009 Dariusz Man et al. This is an open access article distributed under the Creative Commons Attribution License, which permits unrestricted use, distribution, and reproduction in any medium, provided the original work is properly cited.

\section{Introduction}

Organometallic compounds, due to their high biological activity, are the object of intensive research conducted by biologists, geneticists, biochemists, and biophysicists. The research focuses mainly on toxic properties of lead, mercury, and cadmium derivatives [1], that is of metals commonly present in industrial processes of the previous century. However, because of the rapid development of our civilization and global informatization, a fast expansion of the electronic industry generating new technologies is observed, which in turn leads to the appearance of a new source of environment pollution with harmful metal compounds. One of them is tin, popularly applied in the electronic industry and usually treated as a metal of low toxicity. On the other hand, due to its frequent appearance in many products, as well as its ability to accumulate in organisms, tin can become a source of serious poisonings. As it follows from various studies [2-5] organic compounds of tin inhibit, among others, the process of oxidative phosphorylation, accelerate hemolysis of erythrocytes, and also alter the physical properties of lipid model membranes [6, 7]. Ionized forms of tin compounds [8-13] (e.g., chlorides) display a particular activity influencing lipid membranes.
Man [14] reported on a particularly high activity of tin chloride (Triphenyltin chloride-TPhT) built from three aromatic rings and influencing the middle part of lipid bilayer. This compound, after entering the liposomes, considerably fluidizes their structure, which-with reference to biological membranes-means a destructive effect on their functions, that is destruction of cells. It was proved that TPhT inhibits pig testicular 17 beta-hydroxysteroid dehydrogenase activity and suppresses testicular testosterone biosynthesis [15], while in the case of rat's cells-Leydig cells (LC-540) - the compound induces the appearance of apoptosis [16]. Also, triphenyllead chloride used in micromolar concentrations hemolyzes pig erythrocytes [17]. It seems, therefore, purposeful to performe detailed comparative studies of the biological activity of TPhT and its twin lead compoundTriphenyllead chloride (TPhL) in model membranes, as well as in cells of living organisms. Such a comparison can help to understand what function the structure of the molecule plays in the activity of compounds, and what the role of the metal ion transported by this molecule to the membrane is. We also expect to find an answer to the question of what type of interaction, induced by the presence of an admixture in the membrane, decides about its toxic properties. 
Four measurement methods were used in the experiments: two physical ones (the spin probes method and the electric method) in studies of the model membranes, and two biological (minimal inhibitory concentration /MIC/ and test of yeast survival) in studies of the Saccharomyces cerevisiae cells. The influence of TPhT and TPhL compounds on the dynamic properties of liposome membranes formed from Egg Yolk Lecithin (EYL) was investigated by means of the spin probes method (ESR). The electric method was used to examine the transmembrane voltage generated by these compounds on the filters impregnated with an ester of lauric acid. On the other hand, the influence of the examined compounds on living organisms was investigated by means of the MIC method and the test of yeast survival. The yeast Saccharomyces cerevisiae was used as the model organism [18] to investigate the effect of the above-mentioned compounds on the survival of their cells.

\section{Materials and Methods}

2.1. Materials. Saccharomyces cerevisiae wild strain $\mathrm{D} 273-10 \mathrm{~B} / \mathrm{A}_{1}$ was used as the test microorganism. The yeast was grown in YPD medium (1\% yeast extract, $1 \%$ bactopeptone, $2 \%$ glucose). For plating, the medium was supplemented with $2 \%$ bactoagar.

The Egg yolk lecithin (EYL) used in the experiment was prepared at the Institute of Chemistry of Opole University, according to a method described in [19].

The spin probes: 2,2,6,6-tetramethylpiperidine-1-oxyl (TEMPO) and 2-ethyl-2-(15-methoxy-15-oxopentadecyl)4,4-dimethyl-3-oxazolidinyloxy (16-DOXYL-stearic acid methyl ester) as well as the ester of lauric acid were delivered by ALDRICH.

The $50 \mu \mathrm{m}$ thick filters with the pore diameter of $1.5 \mu \mathrm{m}$ used in the experiment were manufactured by Synpor (the Czech Republic).

\subsection{Methods}

2.2.1. ESR Method. The liposomes were formed from EYL in distilled water, in the process of sonification. The total sonification time of a single sample $(1.5 \mathrm{ml})$ lasted to 5 minutes, and the sonification was conducted in consecutive cycles consisting of 30 seconds of sonification followed by 30 seconds of cooling. The concentration of the EYL in the sample was $0.04 \mathrm{M}$ and that of the spin probe (added to the formed liposomes) was $0.05 \%$ in with regard to the lecithin. Two twin compounds, TPhT $\left(\left(\mathrm{C}_{6} \mathrm{H}_{5}\right)_{3} \mathrm{SnCl}\right)$ containing tin, and TPhL $\left(\left(\mathrm{C}_{6} \mathrm{H}_{5}\right)_{3} \mathrm{PbCl}\right)$ containing lead, were chosen for the experiment. The structural formulas of the compounds are presented in Figure 1. Admixtures of the investigated compounds were introduced in the samples after formation of liposomes in a solution of dimethyloformamide (DMF) and their concentrations amounted to $1 \%$ and $10 \%$ (molar) relative to the EYL. The measurements were taken during 180 hours at a constant temperature of $297 \mathrm{~K}$.

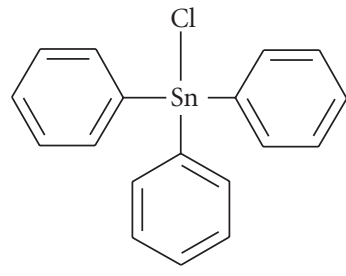

Triphenyltin chloride $\left(\mathrm{C}_{6} \mathrm{H}_{5}\right)_{3} \mathrm{SnCl}(\mathrm{TPhT})$

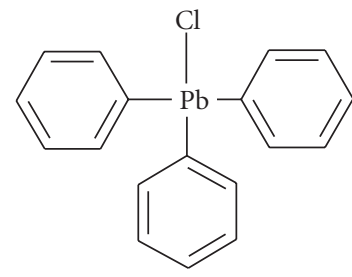

Triphenyllead chloride $\left(\mathrm{C}_{6} \mathrm{H}_{5}\right)_{3} \mathrm{PbCl}(\mathrm{TPhL})$
FIGURE 1: The chemical structure of the TPhT and TPhL molecules.

Two spin probes, penetrating different areas of the membrane, were applied in the experiment: 2,2,6,6-tetramethylpiperidine-1-oxyl (TEMPO) and 2-ethyl-2-(15methoxy-15-oxopentadecyl)-4,4-dimethyl-3-oxazolidinyl-

oxy (16-DOXYL-stearic acid methyl ester). The TEMPO probe dissolves both in the hydrophobic part of liposome membranes and in water environment, whereas the 16DOXYL-stearic acid methyl ester locates deep in the middle part of the hydrophobic layer of the membrane.

On the basis of the ESR spectrum of the TEMPO probe, the spectroscopic parameter $F$ of division of this probe into the membrane and its water surrounding was determined. The measure of parameter $F$ is the ratio of the amplitude $P$ of the high-field line in the ESR spectrum of the probe dissolved in water solution to the amplitude of the low-field line in the lipid environment $H$ (Figure 2(a)). The value of parameter $F$ is connected, among others, with fluidity of the surface layer of membrane [20].

On the basis of the spectrum obtained using the 16DOXYL-stearic acid methyl ester probe, the spectroscopic parameter $\tau$-rotation correlation time-was determined (Figure 2(b)). The value of this parameter also depends on the degree of fluidity of the environment and is the higher; the stiffer (the more ordered) is the environment in which the probe is immersed [21]. In the case of liposomes, this probe locates in the middle of the lipid bilayer and informs about the fluidity of this particular area.

2.2.2. Electric Method. Experimental system consisting of two chambers (the measuring and the reference ones), separated with a membrane (Synpor filter impregnated with lauric acid), was used. The chambers of the measuring system were filled with $10 \mathrm{mM}$ solution of $\mathrm{KCl}$ and connected by means of salt bridges with intermediate chambers, in which measuring electrodes $\mathrm{Ag} / \mathrm{AgCl}$, connected to the electrometer, were immersed. Such a system prevents an interaction that could occur between the measuring electrode and the compound introduced in the chamber. Using the Keithley 6517 electrometer, the time dependence of the transmembrane voltage (TMV), induced by the presence of admixtures of the examined compounds, was measured. Similarly as in the case of the ESR method, the investigated compounds were dissolved in DMF and added-in the same form-to the measuring chamber. The measurements were carried out for two different concentrations of the admixtures: about $1 \%$ and $10 \%$ (molar) relative to the lauric 


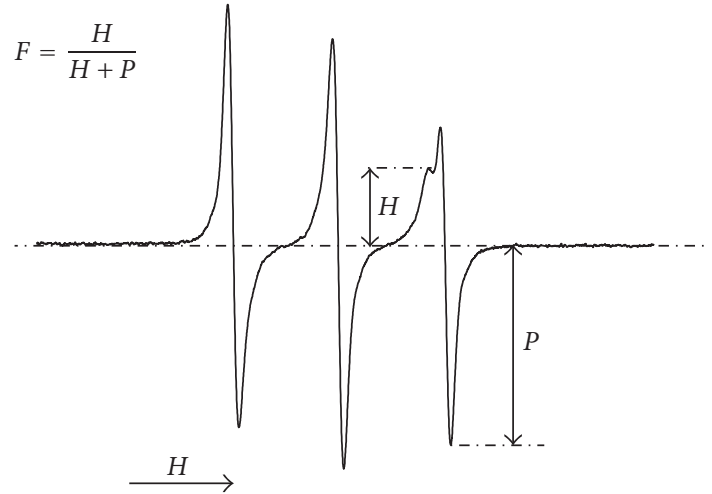

(a)

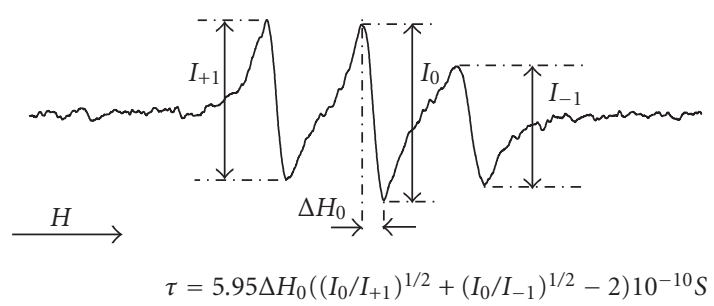

(b)

FIGURE 2: ESR spectra of spin probes placed in the membrane of EYL liposome; (a) TEMPO probe, (b) 16-DOXYL- stearic acid methyl ester.

acid. The concentrations of the admixtures corresponded, approximately, to those applied in the ESR-based measurements.

Each of the ESR and electric measurements was repeated 5 times, and the presented results are the arithmetic means of the measurements. Relative measurement errors amounted to $1.5 \%$ for the parameter $F, 2.5 \%$ for the parameter $\tau$, and $2 \%$ for the electric method, respectively.

2.2.3. Minimal Inhibitory Concentration (MIC) Method. The minimal inhibitory concentration was determined, as previously [22], as the lowest concentration of each compound that inhibits the formation of colonies on YPD plates.

2.2.4. Test of Yeast Survival. The survival test of the yeast treated with the Triphenyltin chloride and Triphenyllead chloride was investigated in liquid YPD medium. Cultures inoculated with $8 \times 10^{2}$ c.f.u. (colony forming unit) were incubated at $30^{\circ} \mathrm{C}$. Samples $(0.1 \mathrm{ml})$ taken at six-hour intervals were plated on the YPD medium, and the viable count was determined.

\section{Results and Discussion}

Figure 3 represents the time dependence of the value of spectroscopic parameter $F$ (Figure 2(a)) of the TEMPO probe introduced in the water suspension of liposomes containing $\mathrm{TPhT}$ and $\mathrm{TPhL}$ admixtures of concentrations $1 \%$ and $10 \%$ in mol proportion to EYL. The broken line in the diagrams marks the values of parameter $F$ for the control sample containing admixture-free liposomes. As it is seen both the TPhT and TPhL admixtures cause a rise in the value of parameter $F$ in relation to the control sample, which can testify to a growth in the fluidity of the surface layer of liposome membranes [20] under the influence of the admixtures. The tin containing compound (TPhT) is more active while interacting with the liposome membranes. However, the influence of concentration of the admixtures on changes in the spectroscopic parameter $F$ is relatively small, which can testify to a weak interaction of the compounds with the surface layer of membranes. In the case of TPhT they amount to $4 \%$ of the value of parameter $F$ in relation to the control sample for a low concentration of the admixture (1\% TPhT in proportion to EYL, Figure 3(a)) and $9 \%$ for a greater concentration of the admixture $(10 \% \mathrm{TPhT}$ in proportion to EYL, Figure 3(b)). The lead containing compound (TPhL) causes even smaller changes in parameter $F$. They amount to about $2 \%$ of the value of parameter $F$ in relation to the control sample for $1 \% \mathrm{TPhL}$ admixture in proportion to EYL and 4\% for 10\% TPhL admixture in proportion to EYL.

Figure 4 shows the time dependence of the value of spectroscopic parameter $\tau$ (rotational correlation time) of the 16-DOXYL-stearic acid methyl ester probe introduced in EYL liposomes (Figure 2(b)) containing TPhT and TPhL admixtures of concentrations $1 \%$ (Figure $4(\mathrm{a})$ ) and $10 \%$ (Figure 4(b)). The broken line in the diagrams (like in Figure 3) marks the values of parameter $\tau$ for the control sample containing admixture-free liposomes. As it follows from the figures, the compound containing tin (TPhT), introduced in the liposomes, causes a decrease in the value of parameter $\tau$ in relation to the control sample, which can testify to a rise in the fluidity of liposome membranes [21] under its influence. On the other hand, the changes in parameter $\tau$, caused by adding a compound containing lead, (both $1 \% \mathrm{TPhL}$ and $10 \% \mathrm{TPhL}$ in proportion to EYL), are small, which can testify to a very weak interaction of this compound with the hydrophobic part of the membrane. The shape of the curves presented in Figures 3 and 4 points to the occurrence of two processes of different dynamics. The first-fast-covers the period from $t=0$ to $t=$ $20-30$ hours and is connected with the effect of admixtures on the liposome membranes. The other process-slowwas extended in time up to about 80-100 hours and was probably connected with gradual hydration and degradation of the sample. After this time no significant changes in the registered parameters $F$ and $\tau$ are observed. A similar dependence was observed in $[13,14,22]$.

Analyzing the changes in the ESR spectroscopic parameters of the TEMPO and 16-DOXYL stearic acid methyl ester probes, it may be concluded as the following.

(1) Despite of the similarity of their structures, the tin containing compound-TPhT-interacts with EYL liposome membranes in their hydrophilic and hydrophobic parts more actively than its lead containing twin-TPhL. Its activity increases with concentration. 


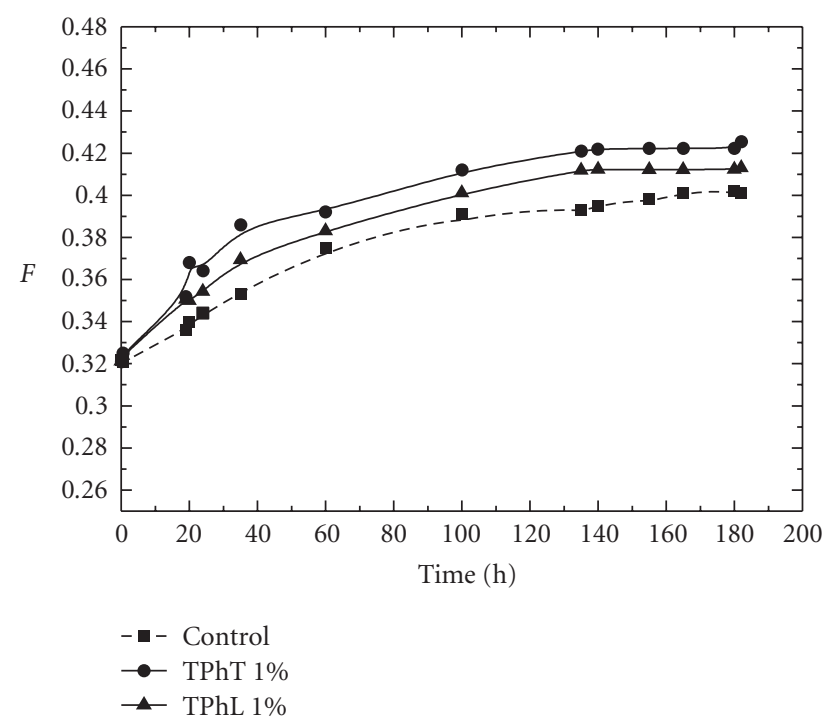

(a)

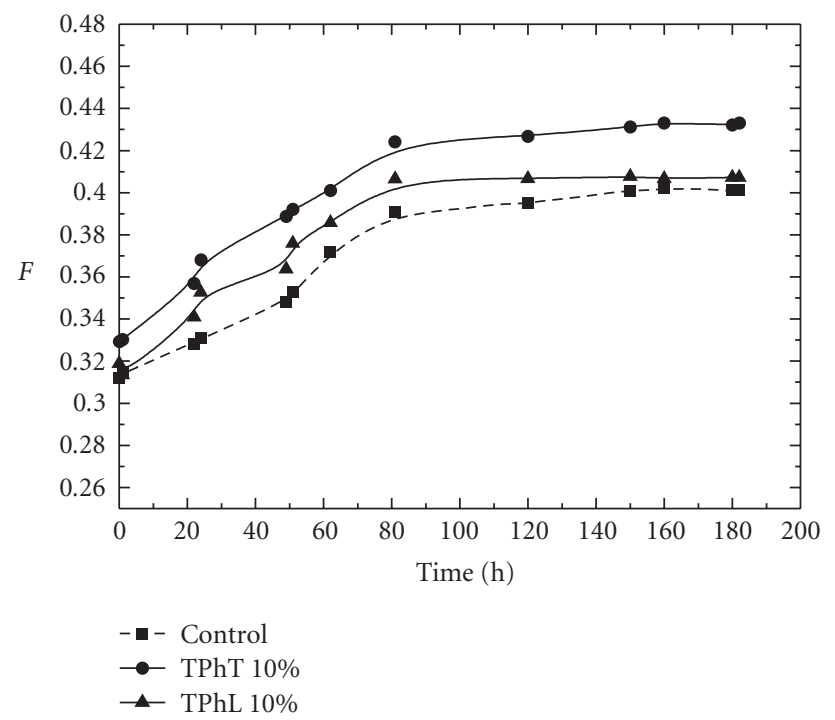

(b)

FIgURE 3: Time dependence of the value of the spectroscopic parameter $F$ of the TEMPO probe, dissolved in water suspension of EYL liposomes containing admixtures of (a) 1\% TPhT and TPhL; (b) $10 \% \mathrm{TPhT}$ and TPhL. The dashed line on diagrams marks the value of parameter $F$ for the control sample containing liposomes without a compound.

(2) The changes in the fluidity of membranes, induced by the presence of TPhT in the hydrophobic area, were decisively greater than those in the hydrophilic one, which can testify to a good penetration of the hydrophobic environment by this compound.

Figure 5 represents the time dependence of the transmembrane voltage (TMV) of filters impregnated with lauric acid, induced by introduction of TPhT or TPhL compounds in the measuring chamber. As it is seen, the transmembrane voltage induced by the containing compound (TPhT) is much higher than that induced by the lead-containing

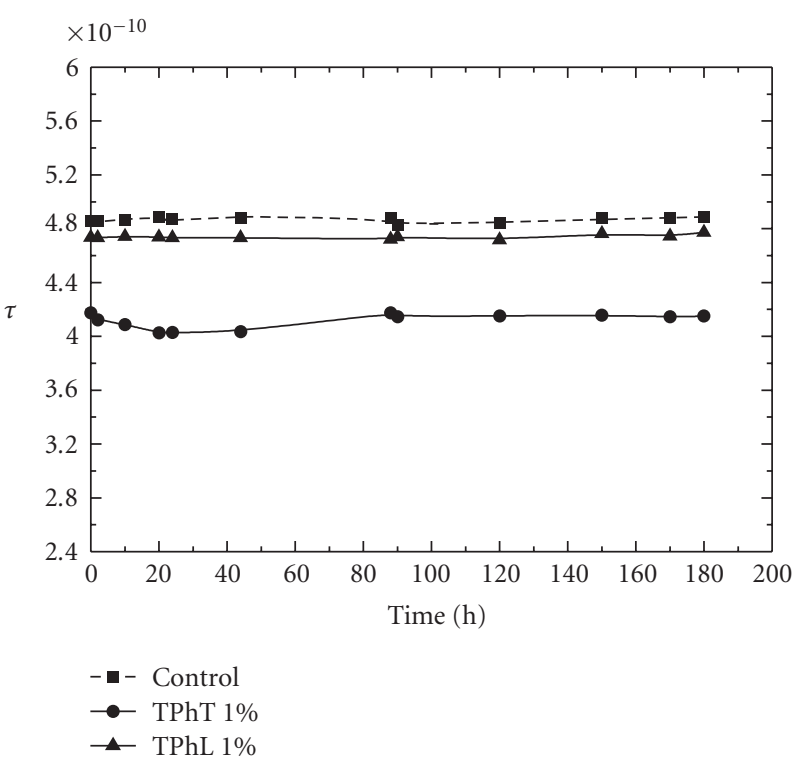

(a)

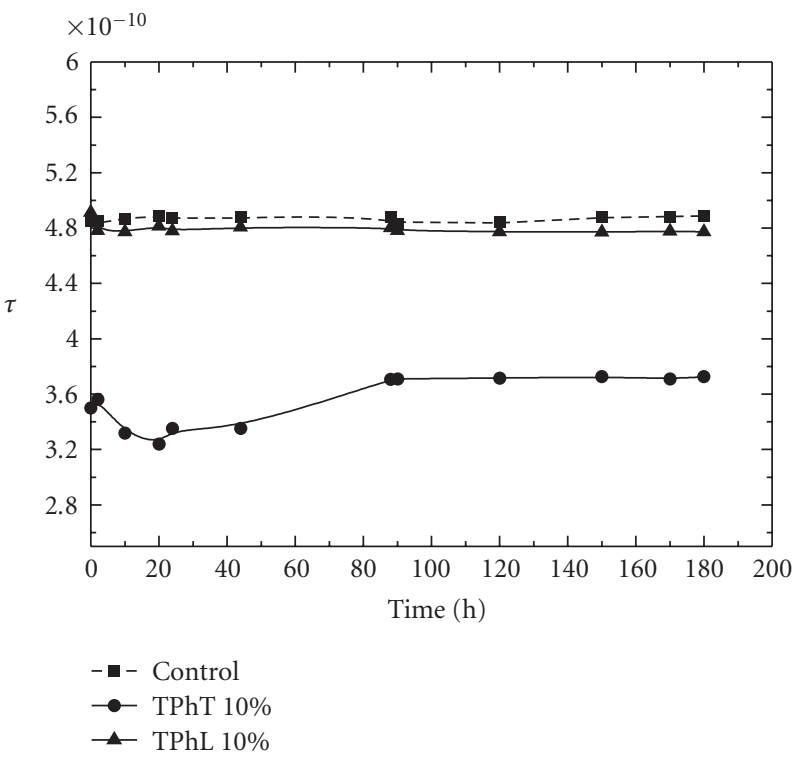

(b)

Figure 4: Time dependence of the value of the spectroscopic parameter $\tau$ (rotation correlation time) of the 16-DOXYL-stearic acid methyl ester probe, dissolved in the EYL liposome membranes containing admixtures of (a) 1\% TPhT and TPhL; (b) 10\% TPhT and TPhL. The dashed line on the diagrams marks the value of parameter $\tau$ for the control sample containing liposomes without a compound.

compound (TPhL). For $1 \%$ concentration of TPhT, the TMV reaches the maximum value of $60 \mathrm{mV}$ after about 90 minutes and then slowly decreases to naught. In the case of TPhL, also for $1 \%$ concentration, the TMV reaches the maximum value of $16 \mathrm{mV}$ after about 30 minutes and in further 330 minutes practically does not change (Figure 5(a)). A rise in the concentration of the examined compounds to $10 \%$ leads to visible changes (Figure 5(b)), as regarding both the shape 


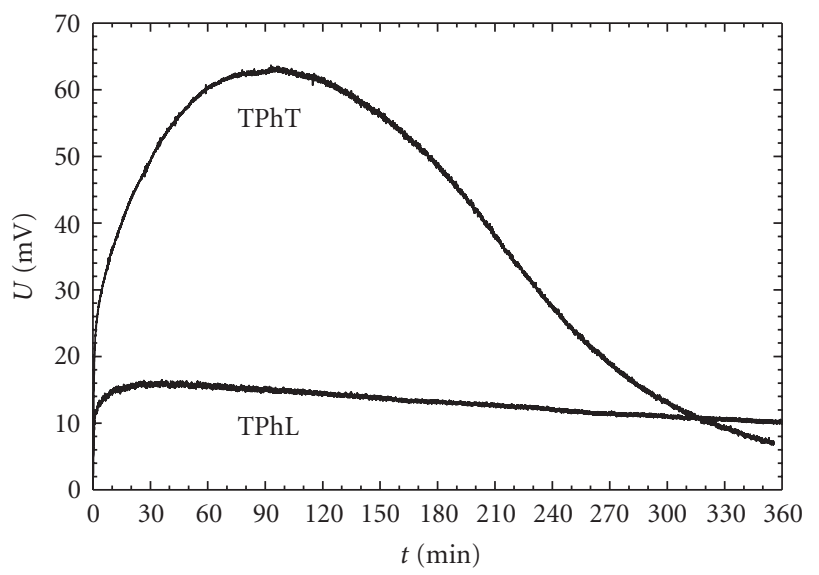

(a)

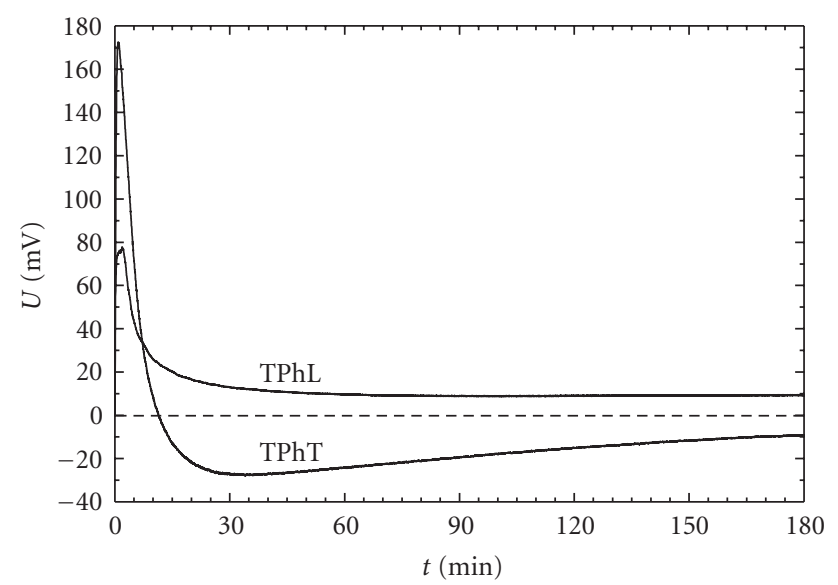

(b)

Figure 5: Time dependence of the membrane voltage for (a) $1 \%$ concentrations of the TPhT and TPhL; (b) 10\% concentration of the TPhT and TPhL admixture in the measurement chamber.

TABle 1: The minimal inhibitory concentration (MIC) of Triphenyltin chloride and Triphenyllead chloride for Saccharomyces cerevisiae growth.

\begin{tabular}{lc}
\hline Compounds & MIC $(\mu \mathrm{g} / \mathrm{ml})$ \\
\hline $\mathrm{Pb}$ compound & 8 \\
Sn compound & 5 \\
\hline
\end{tabular}

of the curves dependence depicting the $U=f(t)$ and the values of the induced TMVs. In the case of TPhT, the TMV reaches the maximum value of $170 \mathrm{mV}$ after about 2 minutes and then-in another 10 minutes-decreases to 0 . After this time the voltage changes its polarization and reaches the minimum value $-30 \mathrm{mV}$, and next it asymptotically increases to about $-10 \mathrm{mV}$. In the case of TPhL, the TMV reaches the maximum value of $80 \mathrm{mV}$ after about 5 minutes and then decreases, and after 60 minutes it stabilizes at the level of $10 \mathrm{mV}$ (Figure 5(b)).

The maxima visible on Figures 5(a), 5(b) testifies to the existence of two competing processes. The first one leads to an increase in the trans-membrane voltage and consists

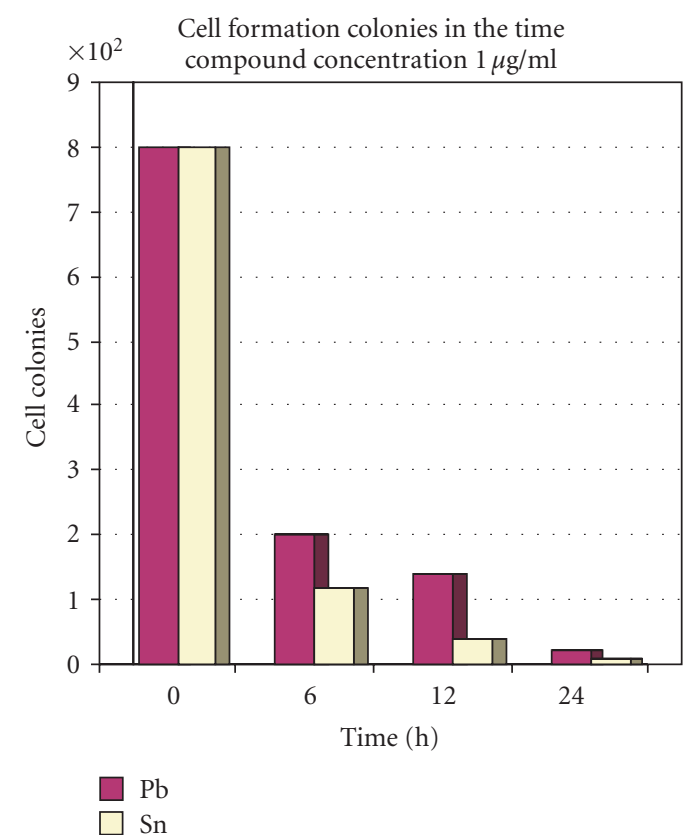

(a)

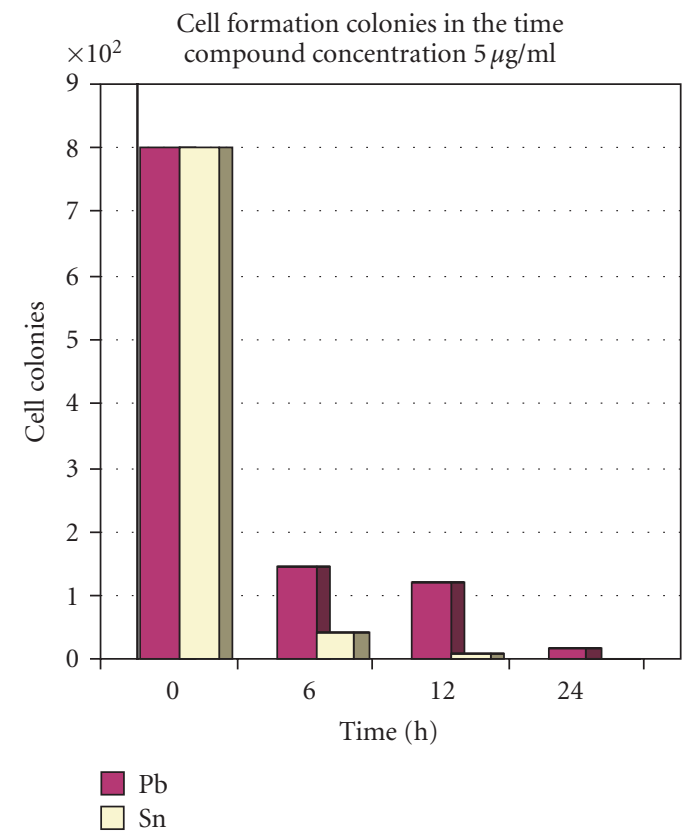

(b)

Figure 6: Time dependence of the concentration of cell colonies containing admixtures of (a) $1 \mu \mathrm{g} / \mathrm{ml} \mathrm{TPhT}$ and TPhL; (b) $5 \mu \mathrm{g} / \mathrm{ml}$ TPhT and TPhL.

in a gradual penetration particles positively charged of admixture dispersed charge in the electrolyte, into the inside of the membrane. This process is additionally stimulated by the negative charge of the membrane (impregnated filter) applied in the experiment. The other process leads to lowering of the trans-membrane voltage by absorption of $\mathrm{Cl}^{-}$ions by the membrane and begins with a delay in relation to the first process, since it requires neutralization of the negative charge of the filter. 
TABLE 2: Dependence of survival of Saccharomyces cerevisiae D273-10B/A doped with Triphenyltin chloride and Triphenyllead chloride on time.

\begin{tabular}{|c|c|c|c|c|c|}
\hline \multirow[t]{2}{*}{ Compounds } & \multirow[t]{2}{*}{ Concentration $(\mu \mathrm{g} / \mathrm{ml})$} & \multicolumn{4}{|c|}{ Formations of cell colonies in time } \\
\hline & & 0 hours & 6 hours & 12 hours & 24 hours \\
\hline Control & 0 & $8 \times 10^{2}$ & $400 \times 10^{2}$ & $250 \times 10^{4}$ & $260 \times 10^{5}$ \\
\hline $\mathrm{Pb}$ & 1 & $8 \times 10^{2}$ & $2.0 \times 10^{2}$ & $1.4 \times 10^{2}$ & $0.3 \times 10^{2}$ \\
\hline compound & 5 & $8 \times 10^{2}$ & $1.45 \times 10^{2}$ & $1.2 \times 10^{2}$ & $0.2 \times 10^{2}$ \\
\hline Sn & 1 & $8 \times 10^{2}$ & $1.18 \times 10^{2}$ & $0.4 \times 10^{2}$ & $0.1 \times 10^{2}$ \\
\hline compound & 5 & $8 \times 10^{2}$ & $0.42 \times 10^{2}$ & $0.1 \times 10^{2}$ & - \\
\hline
\end{tabular}

The investigation of the influence of TPhT and TPhL admixtures on model membranes by means of the electric method confirmed the conclusions arrived at with the use of the ESR method: the tin compound (TPhT) displays a greater activity than the lead-containing compound, TPhL, while interacting with lipid membranes. Both methods confirmed that the organic tin chloride more actively interacts with model membranes than the lead chloride, despite the fact that these compounds have identical chemical structures. An analysis of the changes in the TMVs leads to the following conclusions.

(1) The tin containing compound-TPhT-is more active in interactions with membranes of filters impregnated with lauric acid (similarly as in the case of liposome membranes).

(2) The induced TMVs strongly depend on the concentration of investigated compounds, and-in the case of TPhT at the concentration of $10 \%$-it even resulted in a change in the polarization of the system. The compound containing tin, by heavily fluidizing the structure of the membrane, could cause local micropores to appear in the filter impregnated with lauric acid, which in turn could provoke migration of ions through the hydrophobic inside of the filter toward its other side-a change in the polarization of the registered TMV.

Table 1 represents the results of the MIC test on yeast cells of D273-10B/A strain in the presence of TPhT and TPhL.

The obtained results testify to the fact that both compounds display a strong inhibitory activity; still, it is the tin compound that appears to exert a stronger influence: it is enough to use as little as $5 \mu \mathrm{g} / \mathrm{ml}$ of it to completely inhibit the growth of cells.

The results of the test conducted on the basis of YPD medium in the presence of Triphenyltin chloride and Triphenyllead chloride are presented in Table 2, and their graphical interpretation is shown in Figure 6.

The inhibitory activity of both compounds increases in dependence on the growth time: the longer the cells are grown in the presence of the particular compound, the fewer of them can survive. This experiment points to strong lethal potential of both compounds and, again, the tin compound appears to be stronger as regards its activity in this respect: at a concentration of $5 \mu \mathrm{g} / \mathrm{ml}$, after 24 hours, it causes a complete inhibition of the growth of yeast cells (Figure 6(b)).

\section{Conclusions}

(1) All the measuring methods applied in the present study point to a far more considerable activity of the compound containing tin (TPhT) in its interactions with membranes in comparison with that for compound containing lead (TPhL).

(2) The effectivity of the interaction of the compounds with the membranes increases together with time and with the rise in their concentration, which was confirmed both by the ESR method and the test of yeast survival.

(3) The greater activity of $\mathrm{TPhT}$ in relation to $\mathrm{TPhL}$ is connected with the metal ion inside the molecule, and not with its shape.

(4) The change in the polarization of the registered TMV, induced by the presence of the compound containing tin, can be explained as an effect of strong fluidization of the membrane in the hydrophobic part (results of the ESR method), which could cause local micropores to appear in the filter. This, in turn, induces migration of ions through the hydrophobic inside of the filter to its other side. It is possible that the MIC of $5 \mu \mathrm{g} / \mathrm{ml} \mathrm{TPhT}$ is lethal for the Saccharomyces cerevisiae cells since at this concentration of the compound the cells underwent lysis and as a consequence the membrane loses its structural stability.

The strong influence of the kind of ion enclosed within a large molecule on the biological activity of the latter, when interreacting with both biological and model membranes, seems surprising as well. A similar conclusion can be found in [23], reporting the results of investigations of the family of metalloporphyrins that differed in terms of the ion of metal enclosed inside the molecule. The data presented in [23] demonstrate that the general character of the influence of metalloporphyrins on membranes is very similar (they all fluidize the interior of the membrane), while the effectiveness of their interaction heavily relies on the metal ion. The much higher toxicity of the compound containing tin than that of the one with lead is also quite surprising; the very fact entailing taking a new look at the problem of pollution of environment with metals and forcing us to redefine our views to date on the "low-harmful" tin is present in industrial and communal waste. It may save us, 
in the epoch of common electronization of society, from an unexpected ecological bomb, the more so as other organic tin compounds investigated with the use of the ESR technique [14] also show a considerable activity while interacting with EYL liposome membranes. Comparative studies of these compounds and similar ones containing lead are in progress and their results will be presented in a coming paper.

\section{References}

[1] B. Wiśniowska-Kielian, "Trace element contents in soil and winter wheat-part II: heavy metal contents," Chemia $i$ Inżynieria Ekologiczna, vol. 10, pp. 1021-1030, 2003.

[2] G. Falcioni, R. R. Gabbianelli, A. Santini, G. Zolese, D. Griffits, and E. Bertoli, "Plasma membrane perturbation induced by organotin on erythrocytes from Salmo iridens tront," Applied Organometallic Chemistry, vol. 10, pp. 451-457, 1996.

[3] B. H. Gray, M. Porvaznik, C. Flemming, and L. H. Lee, "Organotin-induced hemolysis, shape transformation and intramembranous aggregates in human erythrocytes," Cell Biology and Toxicology, vol. 3, no. 1, pp. 23-38, 1987.

[4] H. Kleszczyńska, J. Hładyszowski, H. Pruchnik, and S. Przestalski, "Erythrocyte hemolysis by organic tin and lead compounds," Zeitschrift für Naturforschung, vol. 52, no. 1-2, pp. 65-69, 1997.

[5] T. Hamasaki, H. Masumoto, T. Soto, H. Nagase, H. Kito, and T. Toshioka, "Estimation of the hemolytic effects of various organotin compounds by structure-active relationships," Applied Organometallic Chemistry, vol. 9, pp. 95-104, 1995.

[6] A. Miszta, J. Gabrielska, S. Przestalski, and M. Langner, "The effect of phenyltin chlorides on osmotically induced erythrocyte hemolysis," Applied Organometallic Chemistry, vol. 19, pp. 736-741, 2005.

[7] M. Podolak, G. Engel, and D. Man, "Effect of organic tin compounds on electric properties of model membranes," Zeitschrift für Naturforschung, vol. 61, no. 5-6, pp. 453-457, 2006.

[8] M. Podolak, G. Engel, and D. Man, "Interaction of the organic tin chloride with the liquid model membranes," Journal of Physics: Conference Series, vol. 79, Article ID 012038, 2007.

[9] J. Gabrielska, J. Sarapuk, and S. Przestalski, "Role of hydrophobic and hydrophilic interactions of organotin and organolead compounds with model lipid membranes," Zeitschrift für Naturforschung, vol. 52, no. 3-4, pp. 209-216, 1997.

[10] H. Radecka, D. Zieliñska, and J. Radecki, "Interaction of organic derivatives of tin (IV) and lead (IV) with model lipid membranes," Science of the Total Environment, vol. 234, no. 13, pp. 147-153, 1999.

[11] H. Kleszczyńska, J. Sarapuk, and S. Przestalski, "Destabilization of model membranes by organotin compounds," Folia Histochemica et Cytobiologica, vol. 37, p. 31, 1999.

[12] D. Man, M. Podolak, and G. Engel, "The influence of tin compounds on the dynamic properties of liposome membranes: a study using the ESR method," Cellular and Molecular Biology Letters, vol. 11, no. 1, pp. 56-61, 2006.

[13] D. Man and M. Podolak, "Tin compounds interaction with membranes of egg lecithin liposomes," Zeitschrift für Naturforschung, vol. 62, no. 5-6, pp. 427-432, 2007.

[14] D. Man, "Fluidity of liposome membranes doped with organic tin compounds: ESR study," Journal of Liposome Research, vol. 18, no. 3, pp. 225-234, 2008.
[15] S. Ohno, Y. Nakajima, and S. Nakajin, "Triphenyltin and Tributyltin inhibit pig testicular 17beta-hydroxysteroid dehydrogenase activity and suppress testicular testosterone biosynthesis," Steroids, vol. 70, no. 9, pp. 645-651, 2005.

[16] B. Watermann, K. Grote, K. Gnass, et al., "Histological alterations in ovaries of pubertal female rats induced by triphenyltin," Experimental and Toxicologic Pathology, vol. 60, no. 4-5, pp. 313-321, 2008.

[17] H. Kleszczyńska, D. Bonarska, J. Sarapuk, and S. Przestalski, "Protection of erythrocytes against organometals-induced hemolysis," Journal of Fluorescence, vol. 14, no. 1, pp. 5-10, 2004.

[18] A. Goffeau, "1996: a vintage year for yeast and Yeast," Yeast, vol. 12, no. 16, pp. 1603-1605, 1996.

[19] W. S. Singleton, M. S. Gray, M. L. Brown, and J. L. White, "Chromatographically homogeneous lecithin from egg phospholipids," Journal of the American Oil Chemists' Society, vol. 42, no. 1, pp. 53-56, 1965.

[20] E. J. Shimshick and H. M. McConnell, "Lateral phase separation in phospholipid membranes," Biochemistry, vol. 12, no. 12, pp. 2351-2360, 1973.

[21] M. A. Hemminga, "Interpretation of ESR and saturation transfer ESR spectra of spin labeled lipids and membranes," Chemistry and Physics of Lipids, vol. 32, no. 3-4, pp. 323-383, 1983.

[22] E. Obłąk, S. Ułaszewski, and T. M. Lachowicz, "Mutants of Saccharomyces cerevisiae resistant to a quaternary ammonium salt," Acta Microbiologica Polonica, vol. 37, no. 3-4, pp. 261270, 1988.

[23] D. Man, R. Słota, G. Mele, and J. Li, "Fluidity of liposome membranes doped with metalloporphyrins: an ESR study," Zeitschrift für Naturforschung, vol. 63, no. 5-6, pp. 440-444, 2008. 

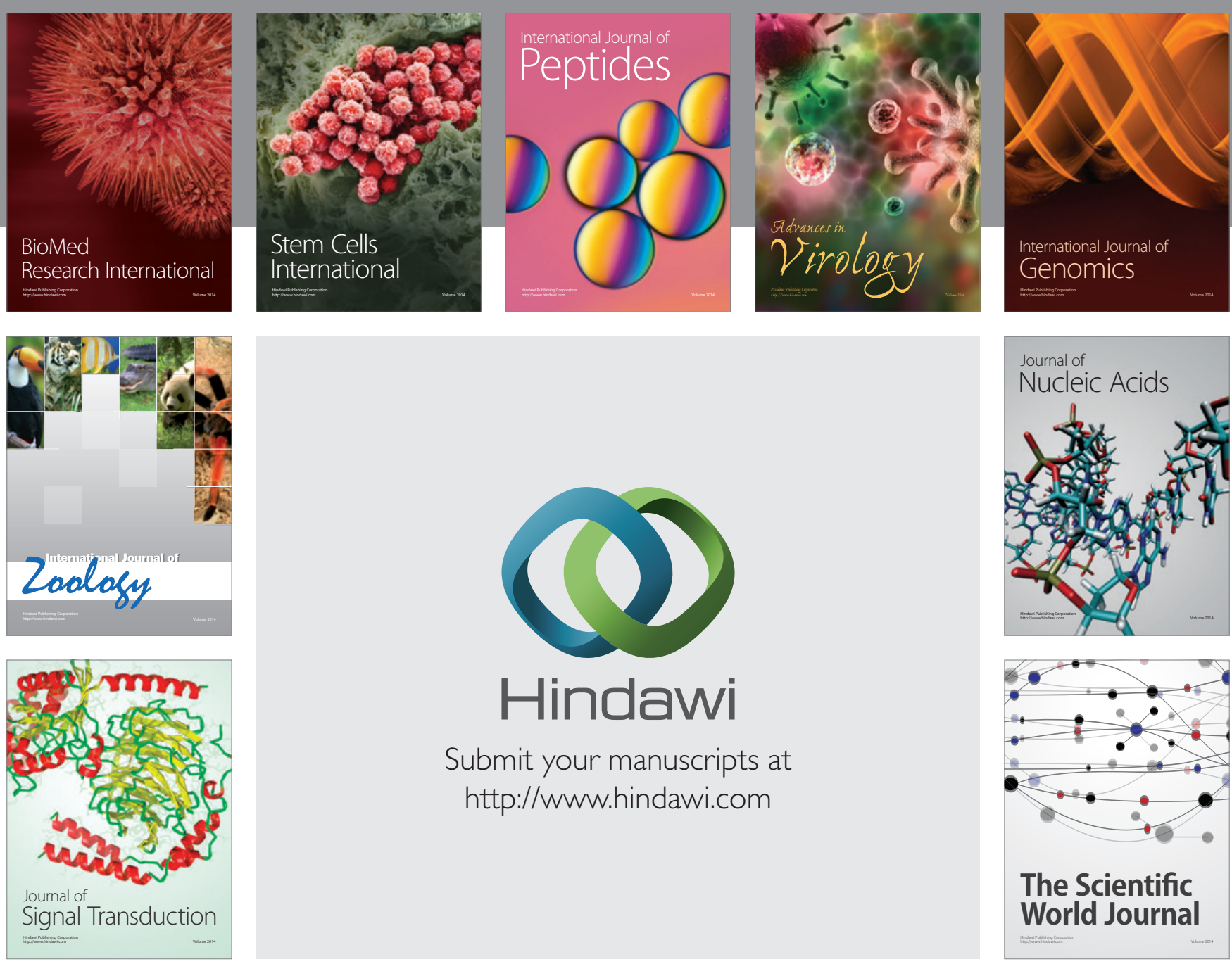

Submit your manuscripts at

http://www.hindawi.com
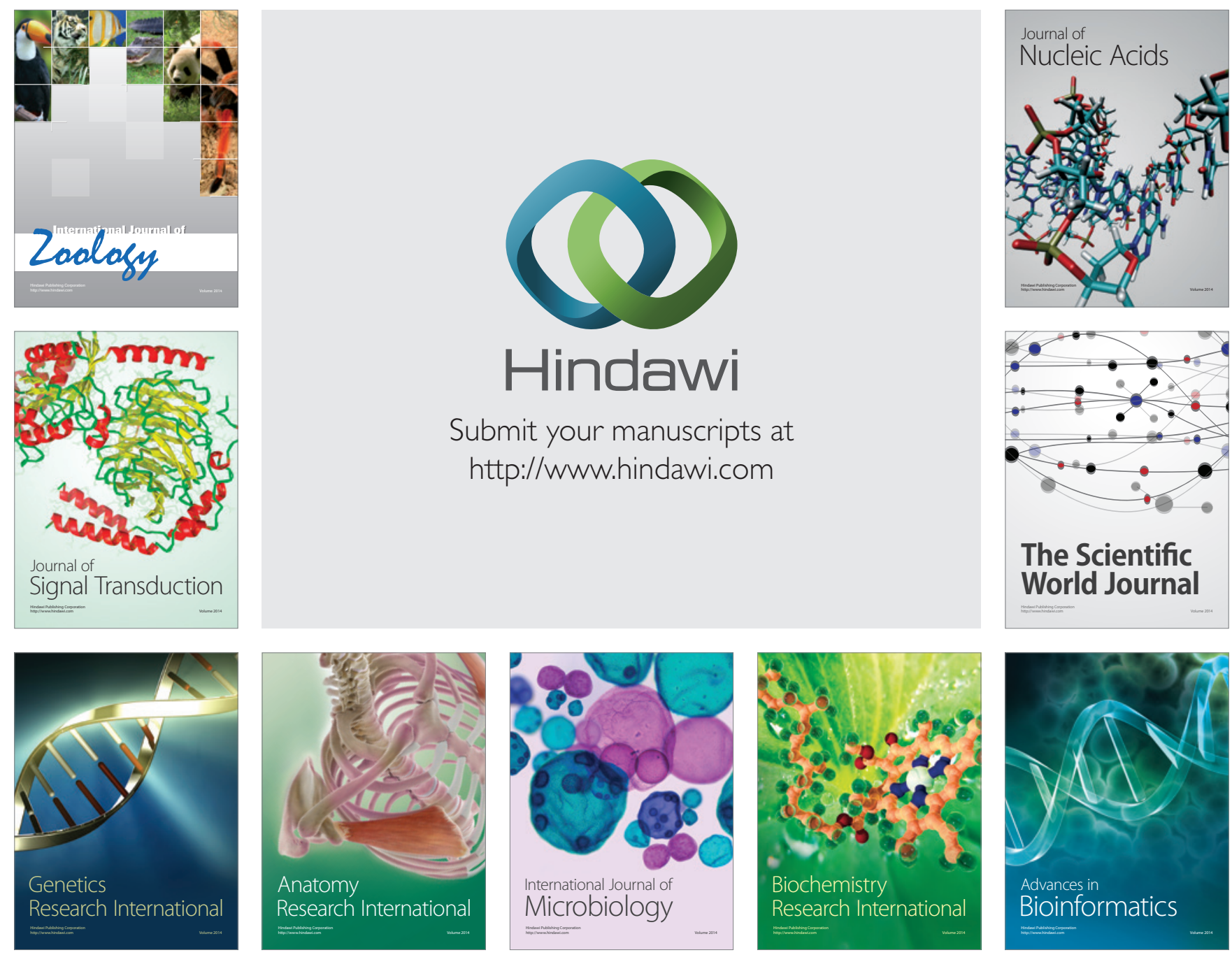

The Scientific World Journal
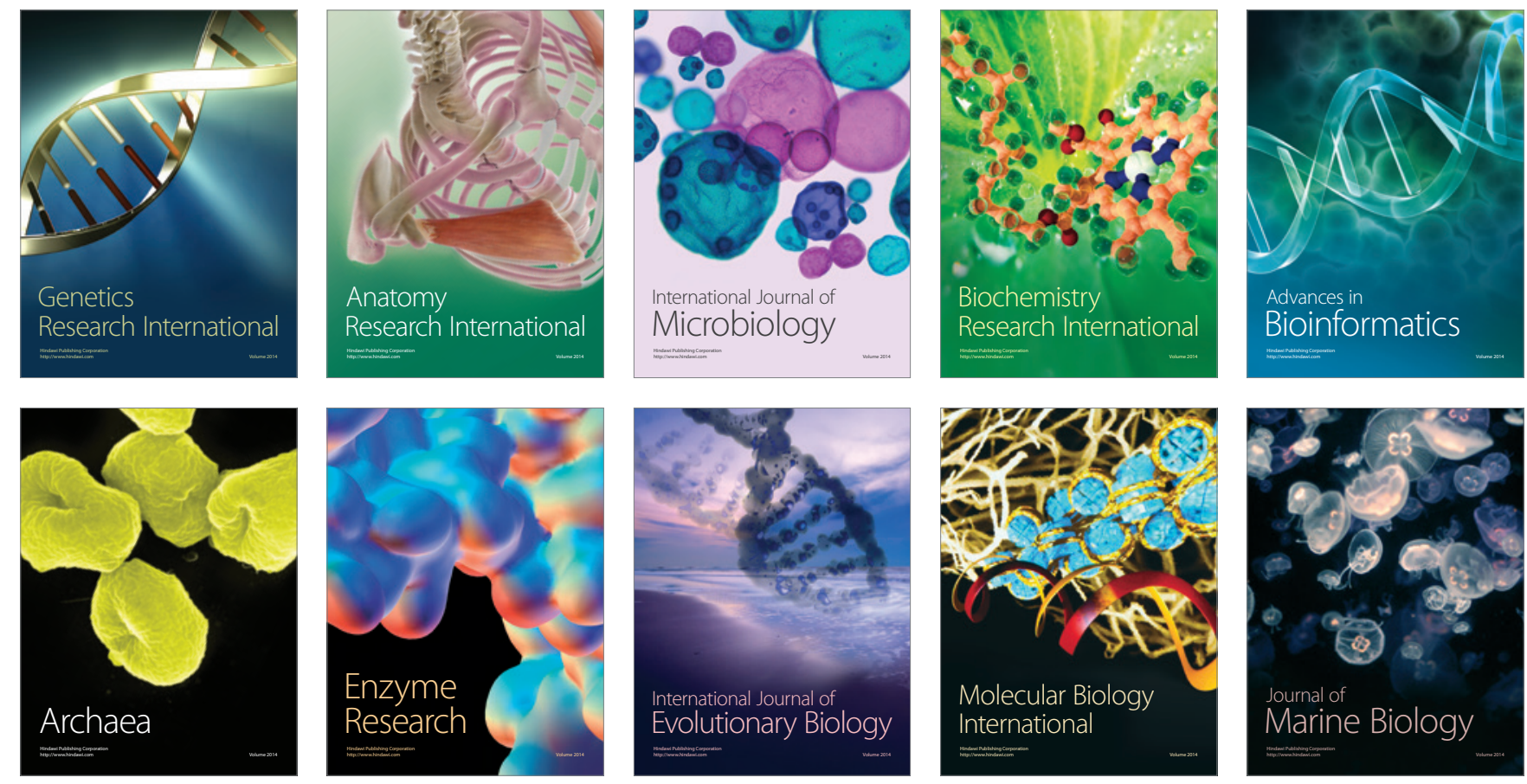